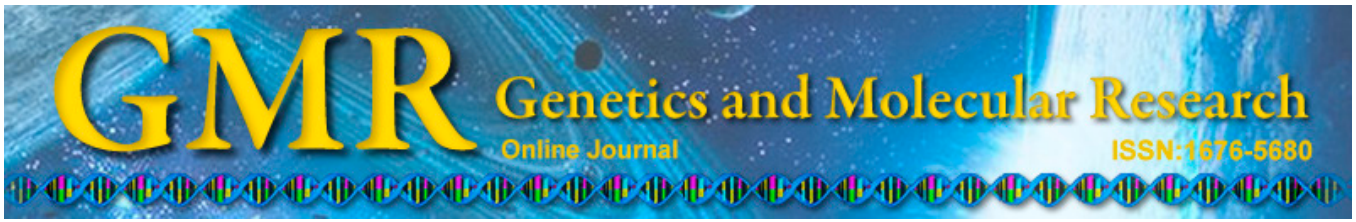

\title{
Gene expression profiles of primary retinal pigment epithelial cells from apolipoprotein E knockout and human apolipoprotein E2 transgenic mice
}

\author{
D.H. Jo ${ }^{1,2,3 *}$, J.H. Lee ${ }^{4 *}$, H.J. Jun ${ }^{4}$, J. Kim ${ }^{1}$, Q. Wen ${ }^{4}$, M.H. Hoang ${ }^{4}$, \\ Y.S. Yu' ${ }^{1,3}$, J.H. Kim ${ }^{1,2,3}$ and S.J. Lee ${ }^{4}$ \\ ${ }^{1}$ Fight Against Angiogenesis-Related Blindness Laboratory, \\ Clinical Research Institute, Seoul National University Hospital, Seoul, \\ Republic of Korea \\ ${ }^{2}$ Department of Biomedical Sciences, College of Medicine, \\ Seoul National University, Seoul, Republic of Korea \\ ${ }^{3}$ Department of Ophthalmology, College of Medicine, \\ Seoul National University, Seoul, Republic of Korea \\ ${ }^{4}$ Division of Food Bioscience and Technology, \\ College of Life Sciences and Biotechnology, \\ Institute of Biomedical Science and Safety, Korea University, Seoul, \\ Republic of Korea \\ *These authors contributed equally to this study. \\ Corresponding author: S.J. Lee \\ E-mail: junelee@korea.ac.kr
}

Genet. Mol. Res. 14 (1): 1855-1867 (2015)

Received February 12, 2014

Accepted August 30, 2014

Published March 13, 2015

DOI http://dx.doi.org/10.4238/2015.March.13.14

\begin{abstract}
Age-related macular degeneration (AMD) causes visual impairment in the elderly. In non-neovascular AMD, studies involving human subjects have suggested potential involvement of aberrant lipid metabolism. However, there have been no reports on gene expression patterns in animal models of non-neovascular AMD
\end{abstract}


with abnormal lipid metabolism such as apolipoprotein $\mathrm{E}$ knockout and human apolipoprotein E2 transgenic mice. Transcriptome analysis was performed using retinal pigment epithelium cells of apoE knockout and apolipoprotein E2 mice using microarray analysis. C57BL/6, Rxrb, Pparbp, Vldlr, and Edf1, which are primarily related to lipid metabolism, were upregulated, while $T g f b r l$ and $P d g f b$, which are related to pathologic angiogenesis in AMD, were downregulated in both types of mice. Apolipoprotein E knockout and apolipoprotein E2 mice showed characteristic gene expression patterns in the transcriptome analysis of primary retinal pigment epithelium cells. These results suggest that specific genes associated with lipid metabolism and angiogenesis are involved in the pathogenesis and progression of AMD.

Key words: Age-related macular degeneration; Apolipoprotein E; Lipid metabolism; Retinal pigment epithelium

\section{INTRODUCTION}

Age-related macular degeneration (AMD) is the leading cause of visual impairment in the elderly (Gehrs et al., 2006). Based on the presence of choroidal neovascularization (CNV), AMD can be divided into 2 types, including non-neovascular (dry) and neovascular (wet) AMD (Jager et al., 2008). Wet AMD is characterized by CNV with disruption of the retinal pigment epithelium (RPE) and underlying Bruch's membrane (de Jong, 2006). Dry AMD is diagnosed when the patient shows drusen and geographic atrophy in the retina (Abdelsalam et al., 1999). Although dry AMD does not result in the formation of subretinal fluid or fibrovascular scar changes, this type of AMD can induce loss of central vision (Christoforidis et al., 2011) and it is clinically important because it can progress to wet AMD.

In the pathogenesis of wet AMD, the main concern involves the pathological angiogenesis of choroidal vessels and disruption of the outer blood-retinal barrier that is composed of RPE (Kim et al., 2006; Jo et al., 2010). In angiogenesis-related blindness of wet AMD, vascular endothelial growth factor (VEGF), platelet-derived growth factor (PDGF), transforming growth factor- $\beta$ (TGF- $\beta$ ), and placenta growth factor have received significant attention (Nagineni et al., 2003; Tolentino, 2009; Hellberg et al., 2010; Jo et al., 2010). In dry AMD, various human studies have suggested the potential involvement of aberrant lipid metabolism in the disease development and progression into wet AMD (Kishan et al., 2011). Lipidic compounds and protein damage caused by lipid peroxidation products are thought to induce RPE dysfunction, which can develop into AMD (Kopitz et al., 2004).

Because apolipoproteinE (apoE) plays a key role in the transportation and metabolism of lipids, apoE is a potential effector in the development of AMD (Bu, 2009). Interestingly, basal laminar deposits were observed in apoE knockout (apoE KO) mice, which are characteristic features of dry AMD (Dithmar et al., 2000). The relationship between apoE isoforms and the AMD development has been examined previously, and we reported that human apolipoprotein E2 transgenic mice (apoE2 mice) showed lipid accumulation and lipid deposits in the RPE (Lee et al., 2007). These results reinforce the potential roles of apoE and specific apoE isoforms in AMD development. 
Although possible associations between apoE and AMD have been demonstrated, there have been no studies examining gene expression patterns in apoE KO and human apoE2 transgenic animal models. We hypothesized that alterations in the gene expression in these animal models of dry AMD would provide insight into the effect of aberrant lipid metabolism during the pathogenesis and development of AMD. In this study, we demonstrated differential gene expression profiles in both animal models compared to in normal controls, suggesting the involvement of a number of genes in the characterization of animal models and the development of non-neovascular AMD.

\section{MATERIAL AND METHODS}

\section{Animals}

ApoE KO and apoE2 mice were purchased from Taconic Farms (Hudson, NY, USA), and C57BL/6 mice were obtained from Orient Bio (Seongnam, Korea) when they were 6 weeks old. ApoE2 mice did not express mouse apoE, but instead expressed human apoE2 (Sullivan et al., 1998). All mice were maintained on regular chow (rodent chow, $4.5 \%$ fat: Purina LabDiet ${ }^{\mathbb{}}$, PharmaServ, Framingham, MA, USA) in a specific-pathogen-free room at $21^{\circ}-25^{\circ} \mathrm{C}$. Water and chow were provided ad libitum. Care, use, and treatment of all animals were conducted in agreement with the ARVO statement for the use of animals in ophthalmic and vision research.

\section{Tissue preparation for electron microscopy}

Eyes were enucleated after sacrificing the mice. For electron microscopy, the enucleated eyes were fixed overnight in a mixture of $2.5 \%$ glutaraldehyde and $2 \%$ paraformaldehyde in $0.1 \mathrm{M}$ phosphate or cacodylate buffer at $\mathrm{pH}$ 7.4. Next, the tissues were fixed for $2 \mathrm{~h}$ in $1 \%$ osmium tetroxide in $0.1 \mathrm{M}$ phosphate buffer. After fixation, the tissues were dehydrated in a graded ethyl alcohol series and propylene oxide and embedded in the epoxy resin (DurcupanACM, Fluka, Buchs, Switzerland). Ultrathin sections were observed under a transmission electron microscope (Jeol, Tokyo, Japan).

\section{Isolation and culture of primary RPE cells}

Mice were fasted for $15 \mathrm{~h}$ before sacrifice. The enucleated eyes were rinsed in $1 \%$ Hank's balanced salt solution (HBSS) containing 1\% penicillin-streptomycin. Next, the eyes were washed in $70 \%$ ethanol and kept in $1 \%$ HBSS for $1 \mathrm{~h}$ at $4^{\circ} \mathrm{C}$. Microdissection of the eye was performed in 1\% HBSS using a Research Stereo microscope (Olympus, Tokyo, Japan) by peeling the sclera and choroid from the posterior segment of the globe. The retina was removed and the sheets of RPE were peeled from the choroid. The RPE sheets were washed in $1 \% \mathrm{HBSS}$ and incubated in $0.05 \%$ trypsin-EDTA at $37^{\circ} \mathrm{C}$ for $30 \mathrm{~min}$. After incubation, the samples were mixed gently by pipetting to separate RPE cells. The suspension was transferred to a conical tube, and then the cells were collected by centrifugation $(415 \mathrm{~g}, 5 \mathrm{~min})$ at $37^{\circ} \mathrm{C}$. Isolated RPE cells were suspended and incubated in Dulbecco's Modified Eagle's Medium (DMEM, Gibco, Grand Island, NY, USA) supplemented with 20\% heat-inactivated fetal bovine serum and $1 \%$ penicillin-streptomycin at $37^{\circ} \mathrm{C}$ in $5 \% \mathrm{CO}_{2}$ atmosphere. The concentration 
of fetal bovine serum was changed to $10 \%$ after 2 passages of the RPE cells. RPE cells from passage 5 to 8 with the confluency of $90 \%$ were used for experiments.

\section{Reverse transcriptase-polymerase chain reaction (RT-PCR)}

Primary RPE cells were seeded at a density of $1.5 \times 10^{5}$ cells $/ \mathrm{cm}^{2}$ on a 6-well plate and cultured for $24 \mathrm{~h}$. Media were then changed to serum-free DMEM and the cells were cultured for additional $12 \mathrm{~h}$. Total RNA was extracted from the cells using TRIzol (Invitrogen, Carlsbad, CA, USA) according to manufacturer instructions. For the synthesis of cDNA, $1 \mu \mathrm{g}$ total RNA was reverse transcribed using oligo $(\mathrm{dT})_{15}$ primer, Superscript II reverse transcriptase (Invitrogen), and dNTPs. PCR was performed with the resulting cDNA templates, 10X PCR buffer, $2.5 \mathrm{mM}$ dNTPs, $10 \mathrm{mM}$ forward and reverse primers, DNA polymerase (Corebiosystem, Seoul, Korea), and sterile water. The primers for mouse $\beta$-actin were 5'-TGCTGTCCCTGTAT GCCTCT-3' (forward) and 5'-AGGTCTTTACGGATGTCAACG-3' (reverse), and the size of the product was 463 base pairs. PCR was started with denaturation in $5 \mathrm{~min}$ at $94^{\circ} \mathrm{C}$, followed by 25 cycles for $30 \mathrm{~s}$ of denaturation at $94^{\circ} \mathrm{C}, 30 \mathrm{~s}$ of annealing at $50^{\circ} \mathrm{C}$, and $30 \mathrm{~s}$ of elongation at $72^{\circ} \mathrm{C}$. The primers for RPE65 were $5^{\prime}$-ATGATCGAGAAGAGGATTGTC-3' (forward) and 5'-CTGCTTTCAGTGGAGGGATC-3' (reverse), and the size of the product was 366 base pairs. PCR was performed with denaturation for $5 \mathrm{~min}$ at $94^{\circ} \mathrm{C}$, followed by 35 cycles for 30 $\mathrm{s}$ of denaturation at $94^{\circ} \mathrm{C}, 30 \mathrm{~s}$ of annealing at $55^{\circ} \mathrm{C}$, and $30 \mathrm{~s}$ of elongation at $72^{\circ} \mathrm{C}$. The $\mathrm{PCR}$ products were electrophoresed on $1 \%$ agarose gels containing ethidium bromide in a constant $100-\mathrm{V}$ field.

\section{Microarray analysis}

Microarray experiments were performed with primary RPE cells from apoE KO and apoE2 mice using the 2-color array system. RPE cells from C57BL/6J mice were used for the control. Total RNA from primary RPE cells was extracted using TRIzol reagent (Invitrogen) and further purified using RNase-Free DNase I (Qiagen, Hilden, Germany) and RNeasyMinElute Cleanup kit (Qiagen). To amplify RNA, the RiboAmp RNA Amplification kit (Arcturus, Carlsbad, CA, USA) was used according to the manufacturer instructions. Total amplified RNA was harvested after 2 rounds of amplification and then additionally purified using the RNaseFree DNase I and RNeasyMinElute Cleanup kit. To synthesize cDNA, reverse transcription was performed with $8 \mu \mathrm{g}$ purified amplified RNA, oligo(dT), Superscript II reverse transcriptase (Invitrogen), and dNTPs. Subsequently, the monofunctional dye Cy3/Cy5 (Amersham Pharmacia, Amersham, UK) was coupled to the cDNA. After the reaction, labeled cDNA samples were purified using the QIAquickPCR purification kit (Qiagen). Next, the cDNA samples were hybridized onto 38.8K mouse exonic evidence-based oligonucleotide arrays (Stanford Functional Genomics Facility, Palo Alto, CA, USA) containing 30,125 constitutive exon probes. The arrays were scanned using a GenePix 4000B (Axon Instruments, Palo Alto, CA, USA), and the data were visualized using the GenePix 4.0 (Axon Instruments).

Probe-level gene expression values were computed with GenePix 4.0 and uploaded to the Stanford Microarray database for normalization and further analysis (Hubble et al., 2009). The data were normalized using NormExp background correction (offset $=350$ ), Loess, and Aquantile methods in the Bioconductor limma package using a fluorescence intensity density plot for 6 array chips of 2 fluorescence channels. Significantly up- and downregulated genes 
were identified by 1 class method of the Significance Analysis of Microarrays (SAM) as implemented in the Bioconductor samr package. The false discovery rate, which was calculated using 500 random permutations of the data set reflecting nonspecific sample variance, was set at 0.0005 for this analysis. Within SAM, comparisons across interventions were analyzed by the Student paired $t$-test by using binary logarithmic $\left(\log _{2}\right)$ expression data of control and treatment groups. We also compared gene expression patterns between RPE cells of two animal models and those of C57BL/6 mice. Biological pathway analysis was assessed using the Database for Annotation, Visualization and Integrated Discovery using commonly up- and downregulated genes in apoE2-KI and apoE-KO mice (Huang et al., 2009a,b).

\section{RESULTS}

\section{Ultrastructure of RPE of apoE KO and apoE2 mice}

We used an electron microscope to examine the RPE of apoE KO and apoE2 mice fed a regular diet. Multiple intracellular lipid deposits were observed in the RPE of both types of mice (Figure 1). The numbers of lipid deposits in the eyes from apoE KO and apoE2 mice were $8.1 \pm 1.5$ and $7.9 \pm 1.1$ per $50 \mu \mathrm{m}^{2}$, respectively. Interestingly, no lipid accumulation was observed in the RPE layer of the normal C57BL/6 mice.
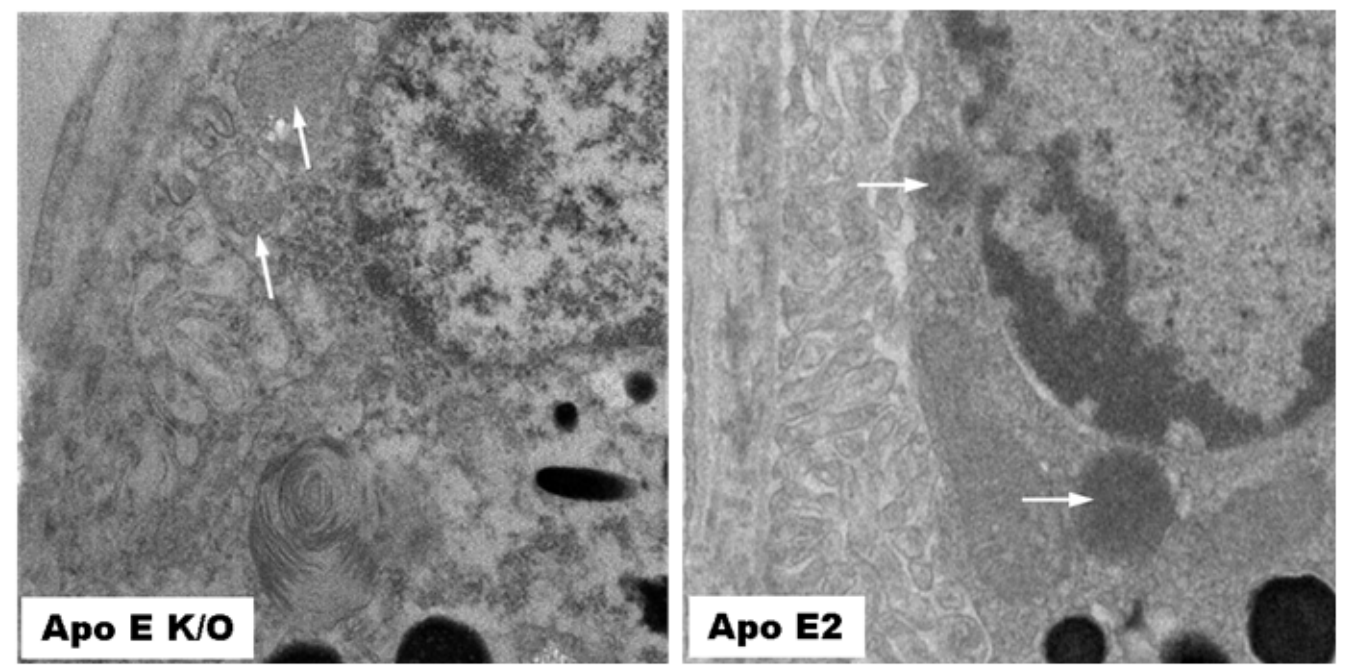

Figure 1. Ultrastructure of eyes in apoE knockout (apoE KO) and human apoE2 transgenic (apoE2) mice was examined by electron microscopy. Eyes from apoE $\mathrm{KO}$ and apoE2 mice on a regular diet showed intracellular lipid accumulation of RPE (white arrows). The numbers of lipid deposits in the eyes from apoE KO and human apoE2 transgenic mice were $8.1 \pm 1.5$ and $7.9 \pm 1.1$ per $50 \mu \mathrm{m}^{2}$, respectively. Interestingly, there was no lipid accumulation identified in the RPE layer of the normal C57BL/6 mice.

\section{Development and identification of the authenticity of primary RPE cells}

Mouse RPE cells were harvested from the eyes of C57BL/6J, apoE KO, and apoE2 mice. RPE cells were successfully developed in DMEM containing $10 \%$ fetal bovine serum 
and $1 \%$ penicillin-streptomycin in $25 \mathrm{~cm}^{2}$ flasks at $37^{\circ} \mathrm{C}$ in a $5 \% \mathrm{CO}_{2}$ atmosphere. Primary mouse RPE cells had an epithelioid appearance (Figure 2A). Additionally, we confirmed the authenticity of primary mouse RPE cells using RT-PCR analysis. The mouse $\beta$-actin and RPE65 genes were clearly expressed in primary RPE cells from C57BL/6J, apoE KO, and apoE2 mice. However, no products were amplified in the human hepatocellular carcinoma cell line HepG2 or the human RPE cell line ARPE-19 (Figure 2B). Primary mouse RPE cells could be maintained up to 8 passages without any undesired effect. For the experiments, we utilized RPE cells from passage 5-8 to induce senescence in the cells (Chen et al., 2010; Liu et al., 2011).

\section{A C57BL/6J}

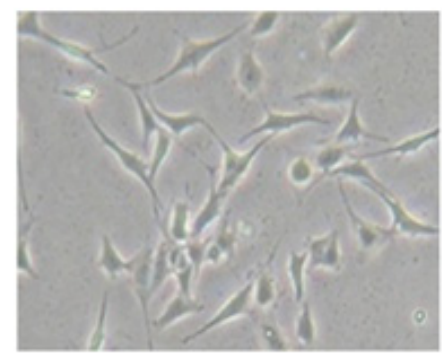

ApoE KO
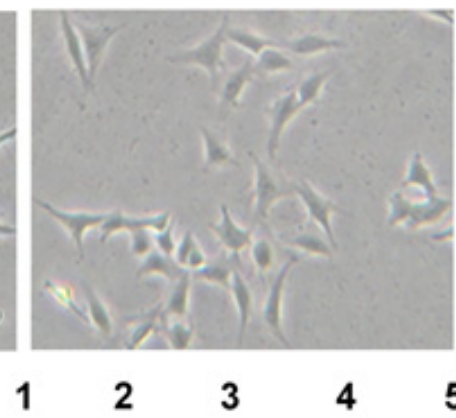

ApoE2

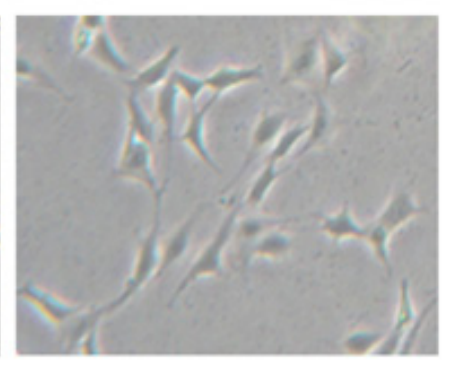

6

B

\section{RPE65}

$\beta$-actin

Figure 2. Primary retinal pigment epithelial (RPE) cells of C57BL/6J, apoE knockout (apoE KO), and human apoE2 transgenic (apoE2) mice. A. Isolated primary mouse RPE cells were developed to have an epithelioid appearance and form a monolayer (magnification, 40X). B. mRNA expression of RPE65 as a mouse RPE marker, and mouse $\beta$-actin as a representative house-keeping gene, in the cells showed the authenticity of primary RPE cells. Lane $1=$ distilled water; lane 2 = HepG2 cells; lane $3=$ ARPE-19 cells; lane 4 = primary RPE cells of C57BL/6J mice; lane $5=$ primary RPE cells of apoE KO mice; lane $6=$ primary RPE cells of apoE2 mice.

\section{Global gene expression profiles in primary RPE cells from apoE $\mathrm{KO}$ and apoE2 mice}

Previously, we reported elevated levels of total cholesterol and triglycerides in apoE2 mice, and Dithmar et al. (2000) observed the same phenomenon in apoE KO mice (Lee et al., 2007). Furthermore, lipid accumulation and basal laminar deposit-like materials were observed in both mice, which are characteristics of dry AMD (Edwards and Malek, 2007). In this regard, we speculated that investigating gene expression profiles from these 2 animal models would enable identification of specific genes involved in AMD with aberrant lipid metabolism. We focused on genes associated with cholesterol metabolism and angiogenesis based on the relationship between both mechanisms and AMD development (Edwards and Malek, 2007; Kishan et al., 2011). 
To investigate differential gene expression patterns in apoE KO and apoE2 mice, we performed SAM analysis using the Bioconductor samr package. A total of 3740 and 4110 transcripts were differentially expressed in the RPE cells of apoE KO and apoE2 mice, respectively. In apoE KO mice, 2861 and 879 transcripts were significantly up- and downregulated, respectively. From the differentially expressed transcripts, selected genes related to the cholesterol metabolism and angiogenesis, including Pparbp (1.61-fold), Ctsb (1.55-fold), Cst3 (1.42-fold), Vldlr (1.24-fold), Esrl (1.24-fold), and Ldlr (1.17-fold), were significantly upregulated, while Tfpi2 (0.92-fold) and $P d g f b$ (0.73-fold) were downregulated (Table 1). In apoE2 mice, 4065 transcripts were up-regulated and 45 transcripts were down-regulated. We focused on genes related to cholesterol metabolism and angiogenesis. The following genes were found to be differentially expressed, including Ctsb (1.48-fold), Rarb (1.4-fold), Esrra (1.39-fold), $r$ Rarg (1.39-fold), Vldlr (1.38-fold), Ahr (1.24-fold), and Esrl (1.06-fold), which were upregulated, and Hprt1 (0.69-fold) and Pdcd4 (0.60-fold), which were downregulated (Table 2).

\begin{tabular}{|c|c|c|c|}
\hline Gene symbol & Gene name & Fold-change & $P$ value \\
\hline \multicolumn{4}{|c|}{ Upregulated genes } \\
\hline Pparbp & Peroxisome proliferator-activated receptor-binding protein & 1.61 & 0.001 \\
\hline$C t s b$ & Cathepsin B & 1.55 & 0.002 \\
\hline Cst 3 & Cystatin C & 1.42 & 0.029 \\
\hline Vldlr & Very low density lipoprotein receptor & 1.24 & 0.013 \\
\hline Esrl & Estrogen receptor 1 & 1.24 & 0.026 \\
\hline Ldlr & Low density lipoprotein receptor & 1.17 & 0.019 \\
\hline \multicolumn{4}{|c|}{ Downregulated genes } \\
\hline Tfpi2 & Tissue factor pathway inhibitor 2 & 0.92 & 0.003 \\
\hline$P d g f b$ & Platelet derived growth factor B polypeptide & 0.73 & 0.003 \\
\hline
\end{tabular}

Table 2. Selected genes associated with lipid metabolism and angiogenesis in the global gene expression profile of human apolipoprotein E2 transgenic mice.

\begin{tabular}{|c|c|c|c|}
\hline Gene symbol & Gene name & Fold-change & $P$ value \\
\hline \multicolumn{4}{|c|}{ Upregulated genes } \\
\hline$C t s b$ & Cathepsin B & 1.48 & 0.025 \\
\hline Rarb & Retinoic acid receptor beta & 1.40 & 0.022 \\
\hline Esrra & Estrogen related receptor alpha & 1.39 & 0.012 \\
\hline Rarg & Retinoic acid receptor gamma & 1.39 & 0.020 \\
\hline Vldlr & Very low density lipoprotein receptor & 1.38 & 0.010 \\
\hline$A h r$ & Aryl-hydrocarbon receptor & 1.24 & 0.026 \\
\hline Esrl & Estrogen receptor 1 & 1.06 & 0.018 \\
\hline \multicolumn{4}{|c|}{ Downregulated genes } \\
\hline Hprt1 & Hypoxanthine guanine phosphoribosyltransferase 1 & 0.69 & 0.009 \\
\hline $\operatorname{Pdcd} 4$ & Programmed cell death 4 & 0.60 & 0.001 \\
\hline
\end{tabular}

\section{Differentially expressed genes from both animal models regarding cholesterol metabolism and angiogenesis}

To identify additional differentially expressed genes, we compared gene expression in animal models with that in normal control C57BL/6 mice. We found that 3374 and 2905 genes 
were significantly upregulated in RPE cells from apoE KO and apoE2 mice compared with those from C57BL/6J mice, respectively. As shown in Figure 3A, 864 genes were upregulated in both mice. Additionally, 371 genes were downregulated in both mice, while 3079 and 1477 genes were significantly downregulated in apoE KO and apoE2 mice, respectively (Figure 3B). Among the commonly regulated genes, we focused on genes associated with cholesterol metabolism and angiogenesis, including Rxrb, Pparbp, Vldlr, and Edf1, which were upregulated, and Tgfbrl and $P d g f b$, which were downregulated (Table 3). Additionally, we performed biological pathway analysis of the commonly up- and downregulated genes. Interestingly, genes involved in primary metabolism (3.61-fold) and vasculature development (2.06-fold) were significantly increased; however, genes involved in lipid biosynthesis (3.01fold) and metabolism (2.01-fold) were decreased (Figure 4).
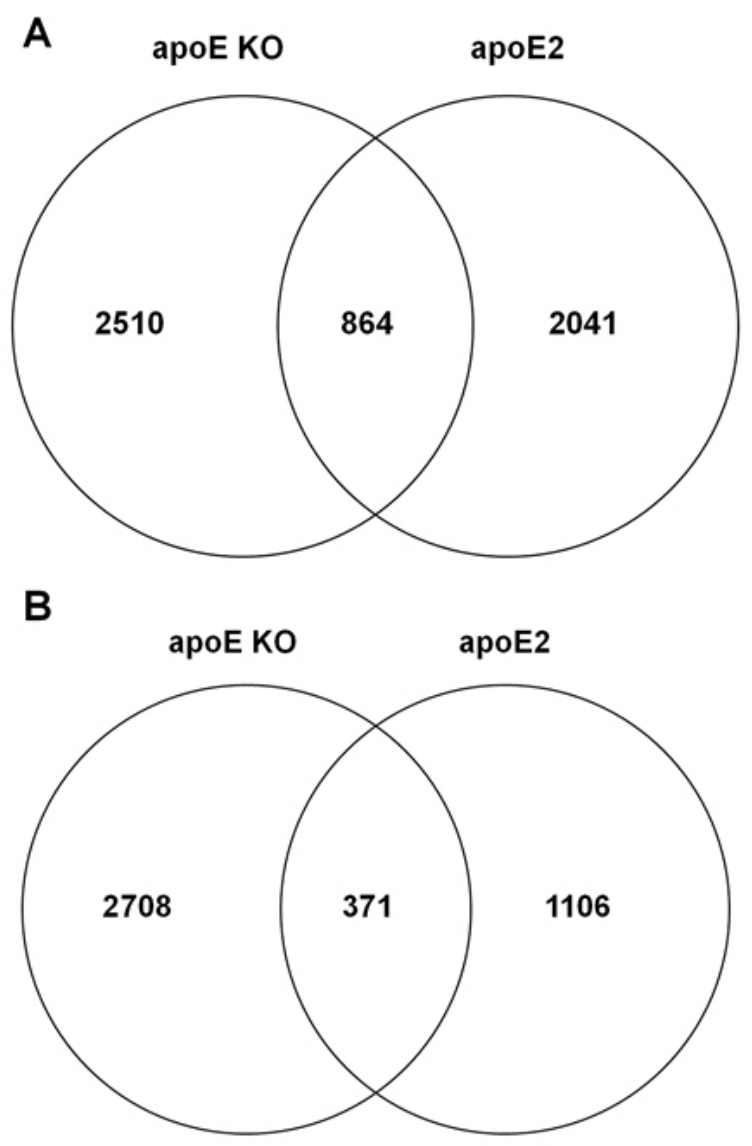

Figure 3. We compared the mRNA expressions from primary retinal pigment epithelium (RPE) cells of apolipoprotein E knockout (apoE KO) mice and human apolipoprotein E2 transgenic (apoE2) mice with those from primary RPE cells of C57BL/6 mice. A. Number of upregulated genes in apoE KO and apoE2 mice compared to C57BL/6 mice. Among the 3374 and 2905 genes that were upregulated in apoE KO and apoE2 mice, respectively, 864 genes were commonly upregulated. B. Number of downregulated genes in apoE KO and apoE2 mice compared to $\mathrm{C} 57 \mathrm{BL} / 6$ mice. Among the 3079 and 1477 significantly downregulated genes in apoE KO and apoE2 mice, respectively, 371 genes were commonly downregulated. 
Table 3. Selected genes associated with lipid metabolism and angiogenesis commonly up- and downregulated genes in the global gene expression profiles of apolipoprotein E knockout and human apolipoprotein E2 transgenic mice.

\begin{tabular}{|c|c|c|c|}
\hline Gene symbol & Gene name & Fold-change & $P$ value \\
\hline \multicolumn{4}{|c|}{ Upregulated genes } \\
\hline Pparbp & Peroxisome proliferator-activated receptor-binding protein & 1.61 & 0.007 \\
\hline$R x r b$ & Retinoid X receptor beta & 1.40 & 0.032 \\
\hline Vldlr & Very low-density lipoprotein receptor & 1.38 & 0.008 \\
\hline$E d f 1$ & Endothelial differentiation-related factor 1 & 1.34 & 0.023 \\
\hline \multicolumn{4}{|c|}{ Downregulated genes } \\
\hline$T g f b r l$ & Transforming growth factor, beta receptor 1 & 0.95 & 0.013 \\
\hline$P d g f b$ & Platelet derived growth factor B polypeptide & 0.78 & 0.011 \\
\hline
\end{tabular}

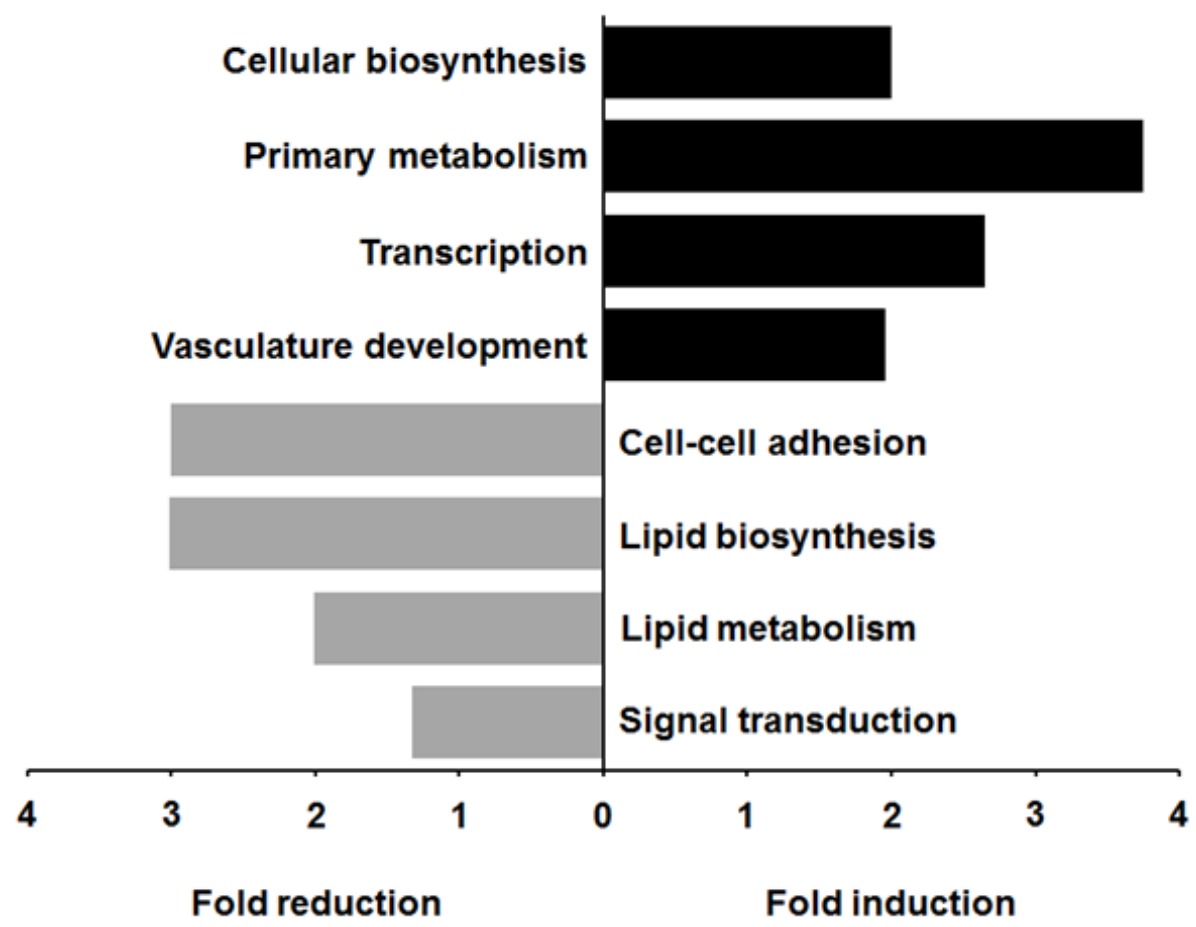

Figure 4. Biological pathway analysis of significantly up- and downregulated genes in apoE KO and apoE2 mice. Pathway analysis was performed using a total of 1235 commonly regulated genes using the Database for Annotation, Visualization, and Integrated Discovery. Five of the most up- and downregulated pathways were presented. The $\mathrm{x}$-axis represents mean fold changes of genes involved in the significant pathways.

\section{DISCUSSION}

We found that Pparbp, Rxrb, Vldlr, and Edf1, most of which are genes associated with lipid metabolism, were upregulated, while $T g f b r l$ and $P d g f b$, which are known to have roles in the formation of pathological angiogenesis in $\mathrm{AMD}$, were downregulated in the gene expression profiles of primary RPE cells from apoE KO and apoE2 mice. Both types of mice 
were used as examples of dry AMD animal models (Dithmar et al., 2000; Edwards and Malek, 2007; Lee et al., 2007). Our study also revealed ultrastructural characteristics of both animal models and increased lipid accumulations in the RPE layer. These results agreed with those of previous studies demonstrating basal laminar deposits and lipid particles in the RPE of both animal models (Dithmar et al., 2000; Lee et al., 2007). Additionally, both apoE KO and apoE2 mice showed hypertriglyceridemia and hypercholesterolemia, suggesting abnormal lipid metabolism (Dithmar et al., 2000; Lee et al., 2007). In this regard, we speculated that investigating gene expression profiles from these two animal models is useful for identifying genetic alterations regarding AMD with aberrant lipid metabolism, as well as for characterizing both animal models.

AMD is one of the leading causes of visual impairment in the elderly (Gehrs et al., 2006). Dry AMD can result in impairment of central vision, and may progress to wet AMD (Christoforidis et al., 2011). In this regard, significant effort has been focused on understanding the pathogenesis of dry AMD, particularly in accordance with dietary fat consumption and lipid metabolism (Kishan et al., 2011). High intake of total fat and trans-fat are thought to be risk factors of AMD (Mares-Perlman et al., 1995; Cho et al., 2001). In addition, in an effort to lower the risk of AMD by lowering serum cholesterol level, multiple studies were conducted using 3-hydroxy-3-methylglutaryl coenzyme A reductase inhibitors, statins (Gehlbach et al., 2009; Ludman et al., 2009).

ApoE serves as a ligand that transports cholesterol and other lipids among various cells of the body through its interaction with apoE receptors (Mahley, 1988). Interestingly, apoE-deficient and apoE2 mice showed characteristics of dry AMD, such as basal laminar deposits and lipid accumulation (Dithmar et al., 2000; Lee et al., 2007). In addition, studies on the association between apoE isoforms and AMD suggested that apoE2 allele predisposed to AMD development and the apoE4 allele had a protective effect against AMD (Fritsche et al., 2009). However, in human studies, the results have been conflicting, and the mechanism of the association has not been determined (Kishan et al., 2011). To determine the biological mechanism, we previously reported increased levels of VEGF and basic fibroblast growth factor (bFGF) in eyes of apoE2 mice (Lee et al., 2007). These results indicate that increased levels of angiogenic factors induce the progression of AMD in the animal model of apoE2 mice, as these two molecules are known to be effectors in CNV development.

This is the first study examining the global gene expression profiles of RPE cells in two different tentative animal models of dry AMD, including apoE KO and apoE2 mice. Our microarray data revealed differential gene expression in both mice. Using SAM analysis, a total of 3740 and 4110 transcripts were found to be differentially expressed in RPE cells of apoE KO and apoE2 mice, respectively. To identify additional differentially expressed genes in apoE KO and apoE2 mice, we directly compared the gene expression profiles between animal models and normal control mice. Interestingly, in pathway analysis of these differentially expressed genes, genes involved in primary metabolism and vasculature development were upregulated. These results agreed with those of our previous study showing an increased level of some angiogenic factors. In contrast, genes involved in lipid biosynthesis and lipid metabolism were generally downregulated. We focused genes that were commonly up- or downregulated, particularly those involved in lipid metabolism and angiogenesis (vasculature development): Rxrb, Pparbp, Vldlr, and Edfl were upregulated, while $T g f b r l$ and $P d g f b$ were downregulated. Interestingly, the former three upregulated were associated with lipid metabolism, while the latter three genes were related to angiogenesis. 
Peroxisome proliferator-activated receptor-binding protein is a key molecule in the function of peroxisome proliferator-activated receptor (PPAR). We speculated that increased expression of the Pparbp gene was associated with abnormal PPAR function. One of the ligands of PPAR is a well-known long-chain polyunsaturated fatty acid, docosahexaenoic acid (SanGiovanni and Chew, 2005). Docosahexaenoic acid also activates other nuclear hormone receptors, such as retinoid X receptor, which is transcribed by the Rxrb (retinoid X receptorbeta) gene. The action of PPAR is thought to maintain endothelial cell function and regulation of VEGF, as well as matrix metalloproteinase-9 (Herzlich et al., 2008). With activation by long-chain polyunsaturated fatty acid, retinoid $\mathrm{X}$ receptor can modulate the induction of the inflammatory signaling pathway, further leading to cell proliferation and production of adhesion molecules in the vascular tissue (Dubuquoy et al., 2002; SanGiovanni and Chew, 2005). Peroxisome proliferator-activated receptor-binding protein and retinoid $\mathrm{X}$ receptor are both affected by specific lipids and play roles in inflammatory cascades related to the pathogenesis of AMD (Herzlich et al., 2008). Endothelial differentiation-related factor-1 (the protein from $E d f 1$ ) is involved in regulating endothelial proliferation and release of nitric oxide (Leidi et al., 2010). Nitric oxide has been studied in the context of oxidative stress in aging (Yu and Yang, 1996); however, an association between endothelial differentiation-related factor-1 and AMD has not been demonstrated.

Interestingly, the Vldlr gene was upregulated in both apoE KO and apoE2 mice compared to in C57BL/6 mice. The very low-density lipoprotein receptor is a member of the low-density lipoprotein receptor family (Herz and Chen, 2006); however, it differs from lowdensity lipoprotein receptor in the cysteine-rich repeat sequences at the $\mathrm{N}$-terminus (Takahashi et al., 2004). Because of this difference, apoE2 binds more easily to the very low-density lipoprotein receptor than to the low-density lipoprotein receptor. Interestingly, Chen et al. (2007) reported that the very low-density lipoprotein receptor may function as a negative regulator of CNV by downregulating the Wnt signaling pathway.

In addition, $T g f b r l$ and $P d g f b$ were downregulated in both types of mice. TGF- $\beta$ and PDGF are known to be involved in the pathogenesis of CNV (Amin et al., 1994; Kliffen et al., 1997; Nagineni et al., 2003). Nagineni et al. (2003) reported that TGF- $\beta$ induced the expression of VEGF in human RPE cells, and in surgically excised CNV membrane from human subjects, there was overexpression of various angiogenic growth factors, including bFGF and TGF- $\beta$ (Amin et al., 1994). In another study conducted by Kliffen et al. (1997), there was significantly increased expression of VEGF, TGF- $\beta$, and PDGF in the retina with AMD. We previously reported that VEGF and bFGF were overexpressed in apoE2 mice, suggesting that by altering other angiogenic cytokines, apoE2 may induce neovascularization (Lee et al., 2007). In fact, there was no evidence of CNV in the eyes of apoE KO and apoE2 mice aged 34-40 weeks. We speculated that upregulation of Vldlr (negative regulator of CNV) and simultaneous downregulation of $T g f b r l$ and $P d g f b$ (active regulators of CNV) may explain why no definite progression from dry AMD to wet $\mathrm{AMD}$ was observed in apoE KO and apoE2 mice.

In summary, this study demonstrated characteristic gene expression patterns in the transcriptome analysis of primary RPE cells of apoE KO and apoE2 mice, two tentative animal models of dry AMD. Specific genes associated with lipid metabolism were overexpressed, suggesting that aberrations in lipid transportation and processing are involved in the development of dry AMD. Interestingly, some genes related with angiogenesis were downregulated. We speculated that downregulation of certain genes involved in angiogenesis caused these animal models to show characteristics of dry AMD, as well as non-progression into 
wet AMD. Based on the present study, the molecules associated with differentially expressed genes should be studied in consideration with the development and progression of AMD, and further studies utilizing these animals as animal models for dry AMD should be conducted to examine these genetic expression patterns.

\section{ACKNOWLEDGMENTS}

Research supported by the Bio-Signal Analysis Technology Innovation Program of MEST/NRF, Republic of Korea (\#2011-0027723), the Mid-Career Researcher Program of MEST/NRF, Republic of Korea (\#2011-0017910), and the Basic Science Research Program of MEST/NRF, Republic of Korea (\#2013R1A2A2A01016176).

\section{REFERENCES}

Abdelsalam A, Del Priore L and Zarbin MA (1999). Drusen in age-related macular degeneration: pathogenesis, natural course, and laser photocoagulation-induced regression. Surv. Ophthalmol. 44: 1-29.

Amin R, Puklin JE and Frank RN (1994). Growth factor localization in choroidal neovascular membranes of age-related macular degeneration. Invest. Ophthalmol. Vis. Sci. 35: 3178-3188.

$\mathrm{Bu} \mathrm{G}$ (2009). Apolipoprotein E and its receptors in Alzheimer's disease: pathways, pathogenesis and therapy. Nat. Rev. Neurosci. 10: 333-344.

Chen Y, Hu Y, Lu K, Flannery JG, et al. (2007). Very low density lipoprotein receptor, a negative regulator of the Wnt signaling pathway and choroidal neovascularization. J. Biol. Chem. 282: 34420-34428.

Chen Y, Wang J, Cai J and Sternberg P (2010). Altered mTOR signaling in senescent retinal pigment epithelium. Invest. Ophthalmol. Vis. Sci. 51: 5314-5319.

Cho E, Hung S, Willett WC, Spiegelman D, et al. (2001). Prospective study of dietary fat and the risk of age-related macular degeneration. Am. J. Clin. Nutr. 73: 209-218.

Christoforidis JB, Tecce N, Dell'Omo R, Mastropasqua R, et al. (2011). Age related macular degeneration and visual disability. Curr. Drug Targets 12: 221-233.

de Jong PT (2006). Age-related macular degeneration. N. Engl. J. Med. 355: 1474-1485.

Dithmar S, Curcio CA, Le NA, Brown S, et al. (2000). Ultrastructural changes in Bruch's membrane of apolipoprotein E-deficient mice. Invest. Ophthalmol. Vis. Sci. 41: 2035-2042.

Dubuquoy L, Dharancy S, Nutten S, Pettersson S, et al. (2002). Role of peroxisome proliferator-activated receptor gamma and retinoid X receptor heterodimer in hepatogastroenterological diseases. Lancet 360: 1410-1418.

Edwards AO and Malek G (2007). Molecular genetics of AMD and current animal models. Angiogenesis 10: 119-132.

Fritsche LG, Freitag-Wolf S, Bettecken T, Meitinger T, et al.(2009). Age-related macular degeneration and functional promoter and coding variants of the apolipoprotein E gene. Hum. Mutat. 30: 1048-1053.

Gehlbach P, Li T and Hatef E (2009). Statins for age-related macular degeneration. Cochrane Database Syst. Rev. 14: CD006927.

Gehrs KM, Anderson DH, Johnson LV and Hageman GS (2006). Age-related macular degeneration - emerging pathogenetic and therapeutic concepts. Ann. Med. 38: 450-471.

Hellberg C, Ostman A and Heldin CH (2010). PDGF and vessel maturation. Recent Results Cancer Res. 180: 103-114.

Herz J and Chen Y (2006). Reelin, lipoprotein receptors and synaptic plasticity. Nat. Rev. Neurosci. 7: 850-859.

Herzlich AA, Tuo J and Chan CC (2008). Peroxisome proliferator-activated receptor and age-related macular degeneration. PPAR Res. 2008: 389507.

Huang da W, Sherman BT and Lempicki RA (2009a). Bioinformatics enrichment tools: paths toward the comprehensive functional analysis of large gene lists. Nucleic Acids Res. 37: 1-13.

Huang da W, Sherman BT and Lempicki RA (2009b). Systematic and integrative analysis of large gene lists using DAVID bioinformatics resources. Nat. Protoc. 4: 44-57.

Hubble J, Demeter J, Jin H, Mao M, et al. (2009). Implementation of GenePattern within the Stanford Microarray Database. Nucleic Acids Res. 37: D898-901.

Jager RD, Mieler WF and Miller JW (2008). Age-related macular degeneration. N. Engl. J. Med. 358: 2606-2017.

Jo DH, Kim JH and Kim JH (2010). How to overcome retinal neuropathy: The fight against angiogenesis-related blindness. Arch. Pharm. Res. 33: 1557-1565. 
Kim JH, Park JA, Lee SW, Kim WJ, et al. (2006). Blood-neural barrier: intercellular communication at glio-vascular interface. J. Biochem. Mol. Biol. 39: 339-345.

Kishan AU, Modjtahedi BS, Martins EN, Modjtahedi SP, et al. (2011). Lipids and age-related macular degeneration. Surv. Ophthalmol. 56: 195-213.

Kliffen M, Sharma HS, Mooy CM, Kerkvliet S, et al. (1997). Increased expression of angiogenic growth factors in agerelated maculopathy. Br. J. Ophthalmol. 81: 154-162.

Kopitz J, Holz FG, Kaemmerer E and Schutt F (2004). Lipids and lipid peroxidation products in the pathogenesis of agerelated macular degeneration. Biochimie 86: 825-831.

Lee SJ, Kim JH, Chung MJ, Wen Q, et al. (2007). Human apolipoprotein E2 transgenic mice show lipid accumulation in retinal pigment epithelium and altered expression of VEGF and bFGF in the eyes. J. Microbiol. Biotechnol. 17: 1024-1030.

Leidi M, Mariotti M and Maier JA (2010). EDF-1 contributes to the regulation of nitric oxide release in VEGF-treated human endothelial cells. Eur. J. Cell Biol. 89: 654-660.

Liu Y, Song X, Zhang D, Zhou F, et al. (2011). Blueberry anthocyanins: protection against ageing and light-induced damage in retinal pigment epithelial cells. Br. J. Nutr. 108: 16-27.

Ludman A, Venugopal V, Yellon DM and Hausenloy DJ (2009). Statins and cardioprotection--more than just lipid lowering? Pharmacol. Ther. 122: 30-43.

Mahley RW (1988). Apolipoprotein E: cholesterol transport protein with expanding role in cell biology. Science 240: $622-630$.

Mares-Perlman JA, Brady WE, Klein R, VandenLangenberg GM, et al. (1995). Dietary fat and age-related maculopathy. Arch. Ophthalmol. 113: 743-748.

Nagineni CN, Samuel W, Nagineni S, Pardhasaradhi K, et al. (2003). Transforming growth factor- $\beta$ induces expression of vascular endothelial growth factor in human retinal pigment epithelial cells: Involvement of mitogen-activated protein kinases. J. Cell Physiol. 197: 453-462.

SanGiovanni JP and Chew EY (2005). The role of omega-3 long-chain polyunsaturated fatty acids in health and disease of the retina. Prog. Retin. Eye Res. 24: 87-138.

Sullivan PM, Mezdour H, Quarfordt SH and Maeda N (1998). Type III hyperlipoproteinemia and spontaneous atherosclerosis in mice resulting from gene replacement of mouse Apoe with human Apoe*2. J. Clin. Invest. 102: 130-135.

Takahashi S, Sakai J, Fujino T, Hattori H, et al. (2004). The very low-density lipoprotein (VLDL) receptor: characterization and functions as a peripheral lipoprotein receptor. J. Atheroscler. Thromb. 11: 200-208.

Tolentino MJ (2009). Current molecular understanding and future treatment strategies for pathologic ocular neovascularization. Curr. Mol. Med. 9: 973-981.

Yu BP and Yang R (1996). Critical evaluation of the free radical theory of aging. A proposal for the oxidative stress hypothesis. Ann. N. Y. Acad. Sci. 786: 1-11. 